\title{
Multiscale Symbolic Transfer Entropy (MSTE) Analysis in Closing Eyes and Being Idle
}

\author{
Lizhao $\mathrm{Du}^{1, \mathrm{a}}$, Wenpo $\mathrm{Yao}^{2, \mathrm{~b}}$, Jun Wang ${ }^{3, \mathrm{c}}$
}

School of Geographic and Biologic information, Nanjing Univ. of Posts \& Telecomm, Nanjing, China a18251955996@163.com, byaowen512@163.com, 'wangj@njupt.edu.cn

Keywords: Multiscale symbolic transfer entropy, closing eyes, idle, EEG

Abstract. It's impressive to learn about how our minds work. In this work, we have discovered that when a person closes his or her eyes, the value of multiscale symbolic transfer entropy(MSTE) [1] of electroencephalogram (EEG, Lead Fp1 and Fp2) grows larger. In other words, the coupling of Lead Fp1 and Fp2 is more significant when the experimenter closes his or her eyes, and we can capture the dynamic information and the its change more easily, which is useful for BCI and other practical uses.

\section{Introduction}

With the fast development of EEG learning, we now can catch the EEG data quickly and analyze it in different ways, such as time domain and frequency domain analysis [2-7], neural network [7-12], chaotic analysis [13-15], etc. In this paper, we use multiscale symbolic transfer entropy (MSTE) to analyze the EEG with Lead Fp1 and Fp2. MSTE, means symbolic the original signal and then calculate its transfer entropy, and finally, try to change the scale factor (for example from 1 to 20) [1]. In this paper, we have extracted 12 sets data when the experimenter closes his or her eyes and the same when he or she is idle. Every sets data is divided into 4 parts, and every part has 5000 samples. The method can be seen in the next part of this paper. The result is that when people close their eyes, the value of MSTE, coupling between Fp1 and Fp2 is larger. This can be helpful to BCI and other practical uses.

\section{Methods}

\subsection{Symbolic}

$S_{i}(\mathrm{i})\left\{\begin{array}{cc}0: & \bar{x}<x_{i+k} \leq \bar{x}+\alpha \times \mathrm{BS}(\mathrm{i}), \\ 1: & x_{i+k}>\bar{x}+\alpha \times \mathrm{BS}(\mathrm{i}), \\ 2: & \bar{x}-\alpha \times \mathrm{BS}(\mathrm{i})<x_{i+k} \leq \bar{x}, \\ 3: & x_{i+k} \leq \bar{x}-\alpha \times \mathrm{BS}(\mathrm{i}),\end{array}\right.$

As the formula [16-18] above, we can change the original data into a symbolic sequence, the result is like the Fig. 1.

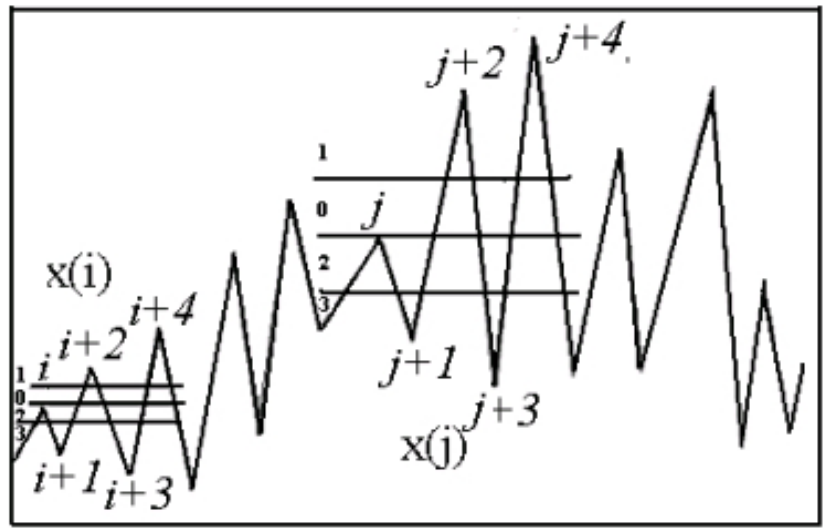

Fig. 1 the result of symbolic sequence

In the formula(1), $x_{i}$ is the $i^{\text {th }}$ data in the original data, $\bar{x}$ and BS(i) respectively denotes the mean and the base scale of the $i^{\text {th }}$ m-dimensional vector $x_{i}$. Here, we choose $\alpha$ as 0.05 , according to the research and experiment we have done earlier. 


\subsection{Transfer entropy [19]}

We then get a symbolic sequence like $1,2,3,3,4,2 \ldots$. In this sequence, count the number of how many time $S_{i}(\mathrm{i})$ has appeared. With Eq. 2, we can calculate the transfer entropy.

$S T E_{X \rightarrow Y}=\sum p\left(x_{t+1}, x_{t}, y_{t}\right) \cdot \log \frac{p\left(x_{t+1}, x_{t}, y_{t}\right) p\left(x_{t}\right)}{p\left(x_{t}, y_{t}\right) p\left(x_{t}, x_{t+1}\right)}$

\subsection{Multiscale [1] analysis}

It is a kind of preprocessing for the original data. With multiscale, we divide the time series into a new one, with Eq. 3.

$y_{j}^{s}=1 / s \sum_{i=(j-1) s+1}^{j s} x_{i}, 1 \leq \mathrm{j} \leq L / s$

Here, $\mathrm{s}$ is the scale factor, and then the length of the new sequence is certain as the L(length of the original time series) divided by s. The basic idea of multiscale analysis is that the system is composed of distinguishable pieces or grains. And here we use coarse-grained process of time series to analyze the EEG data.

\section{EEG data}

This paper uses ECG data extracted from 12 persons when they are idle and closing their eyes. The database has multi-parameter data, including 16 leads. We just extract Fp1 and Fp2 for research. The sampling frequency is $200 \mathrm{~Hz}$.

\section{Experiment results}

The MSTE result is shown in the Fig. 2. Here we can easily learn that the difference is most significant when the scale factor is 16 . With MATLAB, it is verified. Therefore, we select scale factor as 16 for deeper research. And the mean and variance of MSTE value when scale factor is 16 is in the Fig. 3.

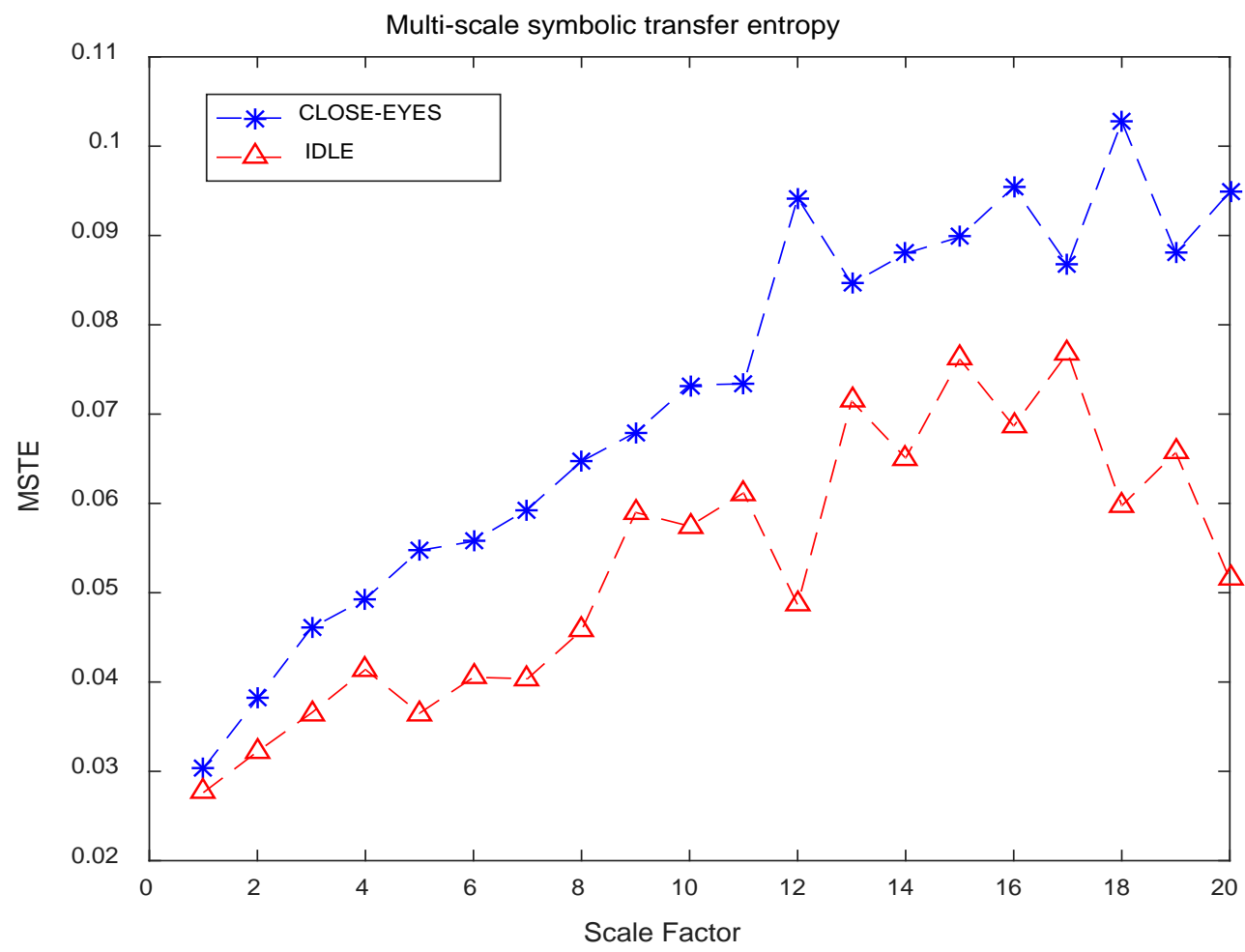

Fig. 2 The MSTE value with changing scale factor 


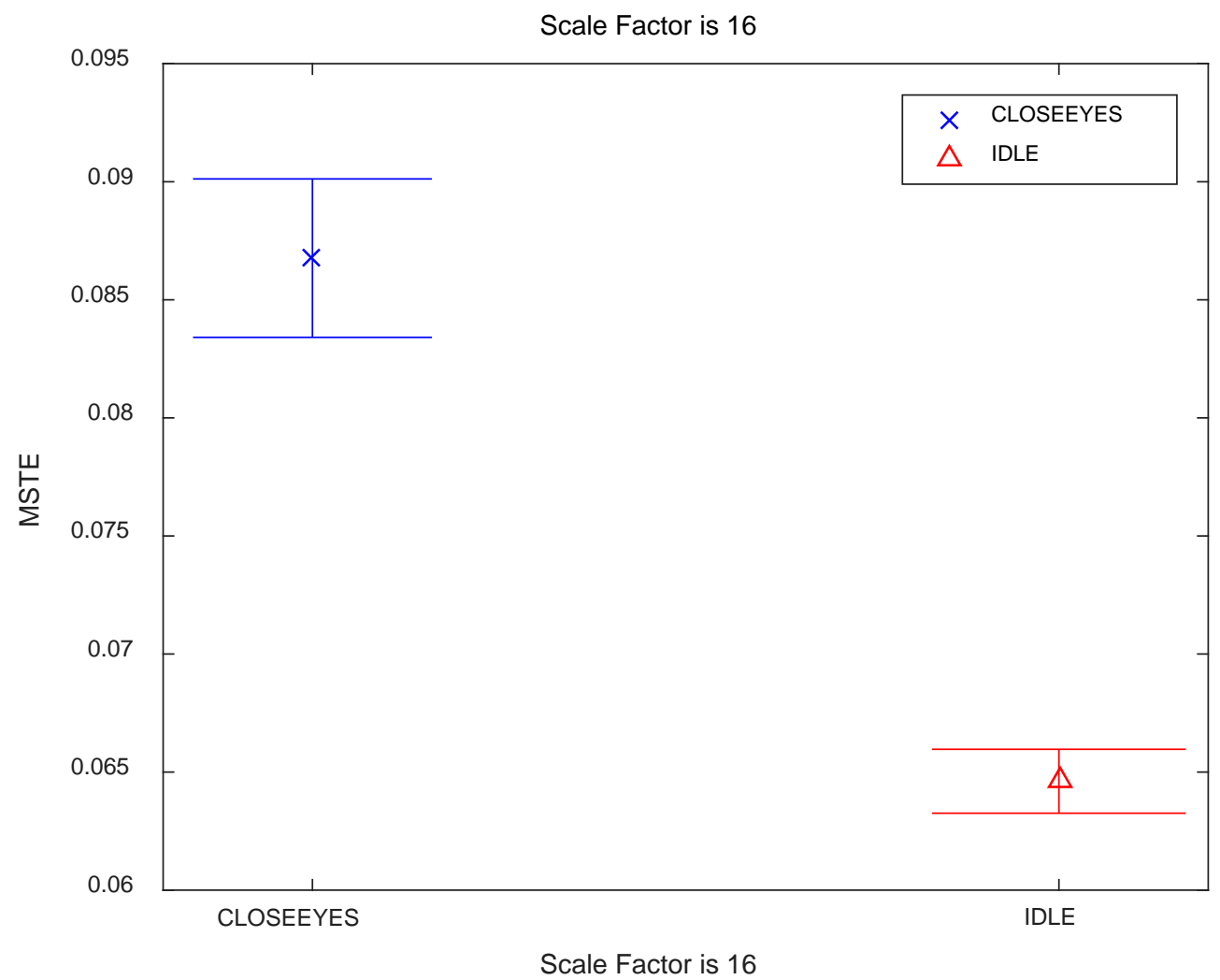

Fig. 3 the mean and variance of MSTE as scale factor is 16

These two figures show that the value of MSTE when people close their eyes is larger, and the mean as well as the variance is also larger. As the scale factor is 16 , the difference is significant, and we can use it for further research. Let's do independent-samples T test for it.

\section{T-Test}

Group Statistics

\begin{tabular}{|rr|l|l|l|l|}
\hline \multicolumn{2}{|c|}{ KINDS2 } & $\mathrm{N}$ & Mean & Std. Deviation & Std. Error Mean \\
\hline VALUE2 & 1.00 & 48 & .08762886 & .062012812 & .008950778 \\
& -2.00 & 48 & .06349738 & .033915386 & .004895264 \\
\hline
\end{tabular}

\begin{tabular}{|c|c|c|c|c|c|}
\hline & & \multicolumn{2}{|c|}{ Levene's Test for Equality of Variances } & \multicolumn{2}{|c|}{ t-test for Equality of Means } \\
\hline & & $\mathrm{F}$ & Sig. & $\mathrm{t}$ & $\mathrm{df}$ \\
\hline VALUE2 & $\begin{array}{l}\text { Equal variances assumed } \\
\text { Equal variances not assumed }\end{array}$ & 5.223 & .025 & $\begin{array}{l}2.365 \\
2.365\end{array}$ & $\begin{array}{l}94 \\
72.807\end{array}$ \\
\hline
\end{tabular}

Independent Samples Test
\begin{tabular}{|ll|l|l|l|}
\hline \multirow{2}{*}{} & \multicolumn{4}{l|}{ t-test for Equality of Means } \\
\cline { 3 - 6 } & & & Std. Error \\
& & Sig. (2-tailed) & Mean Difference & Difference \\
\hline VALUE2 & $\begin{array}{l}\text { Equal variances assumed } \\
\text { Equal variances not assumed }\end{array}$ & .020 & .024131480 & .010201963 \\
& Equa & .024131480 & .010201963 \\
\hline
\end{tabular}




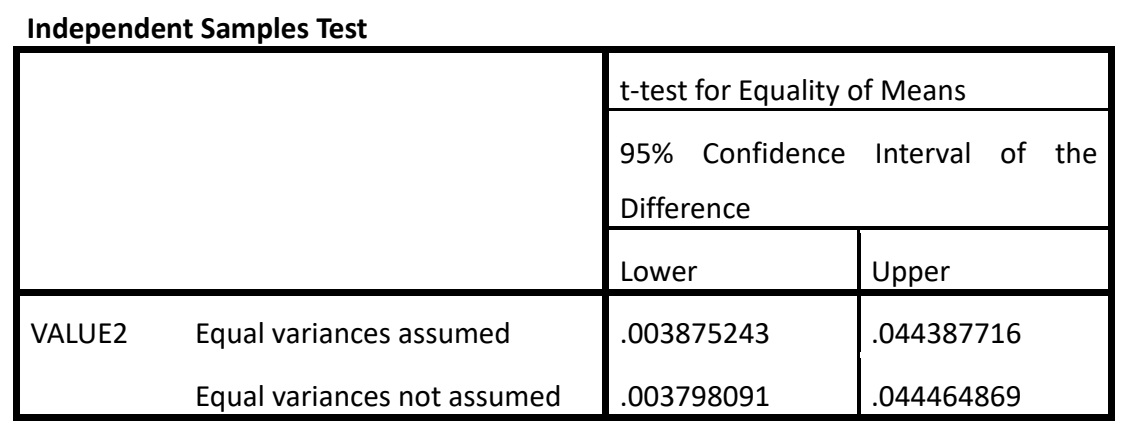

Table 1 the result of independent-samples T test

The result of independent-samples $\mathrm{T}$ test is in the Table 1. Here, we have 12 groups of each data, each has 4 parts, and it is why the $\mathrm{N}$ in table1 is 48 . As $\mathrm{F}$ is 5.223 and the probability is 0.025 , we have to read data in the first line. The result is 0.020 , which means that the result differ significantly. That is to say, when people close their eyes, the value of MSTE varies.

\section{Conclusions}

MSTE is useful and practical in EEG data analysis because it is suitable for dealing with nonlinear time series. In this work, we take it into our researching and only to find that when people close their eyes, the value is larger and the result differs significantly. Although the analysis of EEG is not easy, here we can draw the conclusion that if there are two time series and after calculating their MSTE, the larger would be the one who close his or her eyes or moves. It is to say, when a person makes a move, the value of MSTE would be larger. With these results, we can use BCI to make a choice whether a person moves or not even as closing his or her eyes. Besides, the result is useful in many other fields, for example, medical treatment.

\section{Acknowledgments}

Project supported by the project is supported by the National Natural Science Foundation of China (Grant Nos. 61271082, 61401518, 31671006), Jiangsu Provincial Key R \& D Program (Social Development) (Grant No.BE2015700, BE2016773), the Natural Science Foundation of Jiangsu Province (Grant No. BK20141432),Natural Science Research Major Program in Universities of Jiangsu Province (Grant No.16KJA310002).

\section{References}

[1] Costa M, Goldberger AL, and Peng CK. Phys. Rev. Lett., Multiscale entropy analysis of complex physiologic time series, 2002; 89: 068102-1;

[2] Wang J, Ma Q L 2008 Chin. Phys. B 174424

[3] Hsu W Y 2012 Int. J. Neural Syst. 2251

[4] Petrantonakis P C, Hadjileontiadis L J 2012 IEEE Ton Signal Proc. 602604

[5] Thatcher R W 2012 Dev. Neuropsychol. 37476

[6] Wang J, Yu Z F 2012 Chin. Phys. B 21018702

[7] Musselman M, Djurdjanovic D 2012 Expert Systems with Appl. 3911413

[8] Wang R F, Zhang J H, Zhang Y, Wang X Y 2012 Biomed. Signal Proc. and Control 7490

[9] Orhan U, Hekim M, Ozer M 2012 J. of Med. Syst. 362219

[10] Acharya U R, Sree S V, Alvin A P C, Suri J S 2012 Expert Syst. Appl.39 9072

[11] Siuly S, Li Y 2012 IEEE T on Neur. Sys. and Reh. Eng. 20526

[12] Acharya U R, Molinari F, Sree S V, Chattopadhyay S, Ng K H, Suri JS 2012 Biomed. Signal 
Proc. and Control 7401

[13] Rosenblum M G, Pikovsky A S 2001 Phys. Rev. E 64045202

[14] Smirnov D A, Bezruchko B P 2003 Phys. Rev. E 68046209

[15] Smirnov D A, Bodrov M B, Velazquez J L P, Wennberg R A, Bezruchko B P 2005 Chaos 15 024102

[16] J. Kurths, A. Voss, P. Saparin, A. Witt, H.J. Kleiner, N. Wessel, Quantitative analysis of heart rate variability, Chaos 5 (1) (1995) 88-94.

[17] J. Li, X.B. Ning, Dynamical complexity detection in short-term physiological series using base-scale entropy, Phys. Rev. E (3) 73 (5 Pt 1) (2006) 052902.

[18] N. Wessel, C. Ziehmann, J. Kurths, U. Meyerfeldt, A. Schirdewan, A. Voss, Short-term forecasting of life-threatening cardiac arrhythmias based on

symbolic dynamics and finite-time growth rates, Phys. Rev. E: Stat. Phys. Plasmas Fluids Relat. Interdisciplin. Top. 61 (1) (2000) 733-739.

[19] C. Bandt, B. Pompe, Permutation entropy: a natural complexity measure for time series, Phys. Rev. Lett. 88 (17) (2001) 174102. 